\title{
How Is it a Benefit using Robotic Process Automation in Supply Chain Management?
}

\author{
Elena PUICA \\ Economic Informatics Doctoral School, The Bucharest University of Economic Studies, \\ Bucharest, Romania \\ elenaa.puica@gmail.com
}

Received date: 18 March 2021; Accepted date :19 August 2021; Published date: 19 January 2022

Academic Editor: Anca-Cristina Stanciu

Copyright (C) 2022. Elena PUICA. Distributed under Creative Commons Attribution 4.0 International CC-BY 4.0

\begin{abstract}
The automation of operations within the supply chain management (SCM) is maintained with increased evolution; each part of supply chain management is being affected by the digital transformation and the challenge to become more agile, efficient, and dynamic. To consistently achieve performance, supply chain management (SCM) must focus on highervalue activities that lead to significant results. The automation of low-value activities is important for the continuity and sustainability of supply chain management (SCM) and at the same time for providing better results. Through software robots, omissions that would result from manual processing of SCM activities can be removed. Software robots can exclude downtime by archiving and maintaining a history of actions performed. Software robots work 24 hours/7 days and can be implemented quickly because they can work on the existing architecture of programs that are already used in SCM. Robotic Process Automation (RPA) can be trained and prepared by users to undertake repeatable structured tasks that interact at the same time with multiple computer systems. The article considers the definition of RPA and its benefits in SCM, the use of RPA within SCM, as well as an example of the applicability of RPA in supply chain management.
\end{abstract}

CCS CONCEPTS - Social and professional topics Professional topics Computing and business $\sim$ Automation $\bullet$ Social and professional topics $\sim$ Professional topics $\sim$ Computing and business $\sim$ Computer supported cooperative work.

Keywords: Robotization of Business Processes1; Robotic Process Automation (RPA)2; Software Robots3; RPA in SCM4; RPA Solutions5; Intelligent Process Automation6; Informatic Solutions in SCM7

Cite this Article as: Elena PUICA (2022), "How Is it a Benefit using Robotic Process Automation in Supply Chain Management?" Journal of Supply Chain and Customer Relationship Management, Vol. 2022 (2022), Article ID 221327, DOI: 10.5171/2022.221327 


\section{Introduction}

Technologies are constantly evolving and producing new products and opportunities [1]. In the digital transformation, neither automation nor robotics are new technologies developed. Recently, robotic process automation (RPA) has drawn a lot of attention to the impact it can have on Supply Chain Management (SCM) on automation initiatives. According to the MHI Annual Industry Report (2019) [2], in the top technologies that will impact SCM in the next decade, Robotics and Automation was estimated at $87 \%$. Robots and Automation can improve the overall efficiency of SCM processes by replacing traditional manual tasks, such as sorting, sorting, inspecting, storing, handling, classifying products, and entering data. In a survey, in the Annual Industry Report, MHI talked about the adoption rate of this technology in 2019, the percentage being $34 \%$ and predicted that it will reach $53 \%$ in the next two years and $73 \%$ in the next five years. A motivating factor for SCM to adopt RPA and call on software robots is to perform non-value-added activities scalable and reduce response times [3]. According to the MHI Annual Industry Report (2020), an organization that decided to digitize its SCM processes, the goal was to improve data accuracy throughout the payment claim cycle and replace erroneous manual processes with others automated. Objectives that would be employees to focus on more valuable activities. By adopting RPA, significant improvements in data accuracy have been achieved, reducing manual data entry activities by $65 \%$ to $80 \%$ [7]. RPA offers many benefits for SCM, including faster cycles, improved process reliability/consistency, reduced manual effort, improved data quality and accuracy, time redirected to strategic tasks, increased resource planning flexibility, short path to value. Simultaneously, software robots will, in the future, lead to challenges in providing added value, but also opportunities to do so and create new levels of value [8].

In the literature, there are several works that define, describe, and characterize RPA. There is no standardized definition of RPA.
Fedor Kanakov and Igor Prokhorov noted that "RPA is a modern, innovative technology that allows companies to significantly increase operational productivity by replacing people with software robots, freeing up and redistributing human resources from repetitive, routine tasks to more complex tasks. and interesting, which will add value" [9]. The IEEE Corporate Advisory Group defines RPA as "the use of a pre-configured software instance that uses predefined activities to complete the standalone execution of a combination of processes, activities, transactions, and tasks in one or more software systems to provide a result; or a service" [10]. Madoka et al. describe RPA as an "emerging form of business process automation technology based on the notion of software robots or Artificial Intelligence (AI) workers" [11]. UiPath describes RPA as "a technology that allows anyone to configure computer software or 'robot' to stimulate and integrate the actions of a human who interacts with a computer to complete tasks" [12]. SAP SE describes RPA as "the use of technology to automatically capture and execute applications and processes using software robots" and "the digital transformation of processes by automating tasks and actions that provide no added value" [13]. Van der Aalst et al. describe the RPA as "a general term for tools that operate on the interface of other computer systems as a human would" [14]. Starting from the different descriptions and definitions of RPA in the literature, RPA can be summarized as a technology that allows process automation through a sequence of pre-configured activities that aims to replace routine and repetitive activities performed by human resources.

The article considers the definition of Robotic Process Automation (RPA); in the first section are presented the benefits of RPA in SCM; in section two the use of RPA within SCM is provided; lastly some examples of the applicability of RPA in SCM are given. The article ends with the main conclusions of the use of RPA in SCM. 


\section{Benefits of RPA In Supply Chain Management}

RPA offers many benefits in general, but also in the field of SCM, which has the overall objective to provide, rather, the shortest path to automation by integrating software robots with the user interface, rather than interacting with the application code. RPA can also help manage SCM by solving systematic automation challenges based on repetitiveness. The main benefits of the RPA technology highlighted by Buccowich are accuracy, improved employee morale, productivity, reliability, consistency, noninvasive technology, compliance, and a low technical barrier [15]. Alisha Asquith and Graeme Horsman talked about the following benefits of RPA: coherence, cost, compatibility, scheduling time, the need for good organization and consistency [16].

Some important benefits of RPA in SCM:

a. Accuracy: RPA can significantly increase the accuracy of SCM operations because it is less prone to procedural errors. Any robot that is programmed correctly and has its functionality tested and verified will perform the task with consistent assurance. Any modification of the variable results in the failure to complete a process, marking notable errors to the user, indicates that a process was not completed as expected.

b. Consistency: RPA can automate any SCM business process that can be repeated and rule-based, with extreme precision at a very high processing speed. Robots can only perform preprogrammed commands; unexpected deviation of the load is not possible. This is an important factor as it allows you to check any results.

c. Compliance: RPA "robots" comply with the rules of compliance with regulations because they can only operate through a succession of actions. If a company has a policy or standard that must be followed when conducting an action, RPA technology can comply with that standard. It can provide a history of audit trails of actions taken. RPA allows the user to find problems faster and meet stricter standards of compliance. In the context of SCM, this means that, upon completion, the actions of a robot can be verified to determine new elements of successful business improvement [22].

d. Compatibility: Any robot must be able to interact with the components of an interface, but the ability to interact depends on how the interface is programmed. Compatibility provides a barrier to RPA adoption in SCM, where the impact of adoption is difficult to ascertain due to limited available information. Automation reduces work time/tasks and increases productivity. In this context, SCM organizations can move to RPA compatible platforms.

e. Programming time: Before RPA can begin providing procedural benefits, functionality must be programmed. This means that an organization must have the relevant internal expertise to do so as well as the ability to identify appropriate areas for robot application.

f. The need for a good organization and consistency: RPA is procedural in nature and therefore any SCM must allow for good and consistent organization within their digital structures. If the file paths and naming conventions of the relevant content are non-compliant, it is more difficult for robots to be programmed in a way that allows tasks to be repeated without procedural errors. Therefore, a strong emphasis is placed on determining and implementing a good organizational activity.

\section{Using RPA In Supply Chain Management}

In SCM, especially in the retail industry, many processes are used that are performed manually, constantly looking for technologies to improve accuracy and productivity. The use of software robots can help automate various retail processes, including providing customer support. Some examples of situations in which by 
using RPA and using this technology can demonstrate efficiency in SCM processes are tracking orders and providing necessary updates to customers at predetermined intervals. Also, automating the collection of customer feedback increases customer satisfaction. Efficient automation allows for return management, inventory adjustment and the customer-lending process. It can also help automate many manual tasks in accounting, including debt, receivables, reconciliation, financial closing, financial recording, etc. RPA can be used in various analysis functions, including campaign analysis, consumer behavior analysis, etc. In addition, RPA can automate the collection of planning data, clean up data, run simulations, identify exceptions, and communicate plan data to requested stakeholders.

In logistics and supply chain, RPA can be used to monitor the inventory process, track shipment information, and communicate with customers/suppliers based on pre-configured events. RPA offers the possibility to automate various use cases in inventory management, merchandise management and supplier management. For logistics, RPA can automate transport management, effectively monitoring goods in transit. RPA can also provide valuable information regarding carriers, insurance companies based on the statistical data set. In production, technologies are constantly being sought to improve the processing speed, scalability and to simplify manufacturing operations. The use of software robots can help the manufacturing industry in various processes. RPA can help in the efficient management of information from the "list of materials" data, including raw materials, components and subcomponents that create new products. It can also help resolve low-priority customer queries by entering necessary data from various sources and can facilitate automatic email communication with users. Enterprise Resource Planning (ERP) automation can facilitate the generation of various reports, including debits, receivables, pricing, etc. RPA can also automate tasks between ERP functions, which are not limited to inventory management, supplier management, customer management, debt, accounts receivable, General Ledger (GL), etc. RPA can help digitize and migrate information from old data sources into an ERP system with a high level of accuracy.

RPA can provide detailed statistics of SCM processes, including successful execution, determination of process bottlenecks and possible exceptions. This valuable perspective can help refine manufacturing processes. Figure 1. shows the level of usability of RPA in SCM between 20162020. 


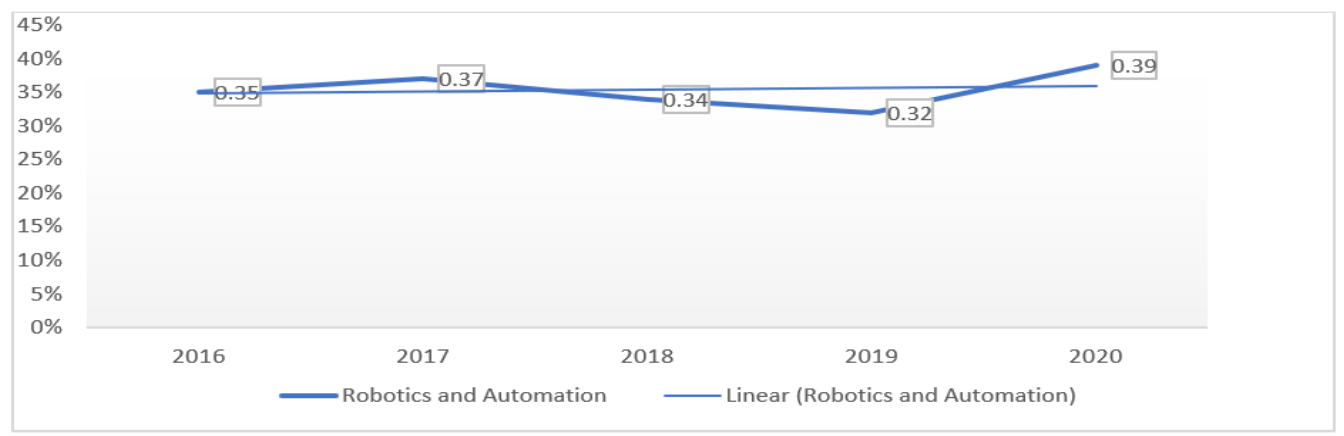

Fig. 1: The usability level of Robotics and Automation in Supply Chain Management (2016 - 2020). Author's own processing based on data provided by MHI Annual Industry Report. (https://www.mhi.org/publications/report\#download)

The use of robots and automation of activities in the procurement and logistics chain in the last four years has had an increased pace in terms of its use in supply chain management. 2017 compared to 2016 had a growth rate of the use of this technology by $5.71 \% .2018$ compared to 2017 , the use of this technology decreased by $8.11 \%$. 2019, compared to the previous year (2018) in which it decreased by $5.88 \%$. 2020, compared to 2019, the usability rate of this technology increased by a percentage of $21.88 \%$. In total, comparing the four years (2016-2020), the usability rate of automation and robotics technology is increasing with a percentage of $11.43 \%$.

\section{Example of use of RPA in SCM}

The implementation of software robots in SCM requires a costly manual analysis of tasks performed by users, once, by observing their behavior, which is not amplified when there are hundreds of processes or by a careful analysis of process documentation, which could be possible to be out of date. Finding opportunities to automate activities more complex than routine repeat activities requires the use of structured and unstructured data from process procedures. There have been several research efforts to identify automation activities [17] [18] in textual descriptions, but they focus on certain areas of business (example, utility bill generation). Identifying activities that can be automated solves only part of the problem. The results should also be complemented by Artificial Intelligence (AI) models, which are appropriate for automating activities [19]. In many cases in SCM, the adoption of RPA tends to focus on automating activities and not on automating processes. This approach gives a higher value, but also has the disadvantage that it may not meet the expectations that tend to occur. A challenge when choosing to implement software robots is the need to discover and analyze the processes that are appropriate for automation. This approach leads to a greater awareness of the relationship between software robots and other process-focused tools, such as process extraction and discovery, process design, process integration, and process monitoring. 


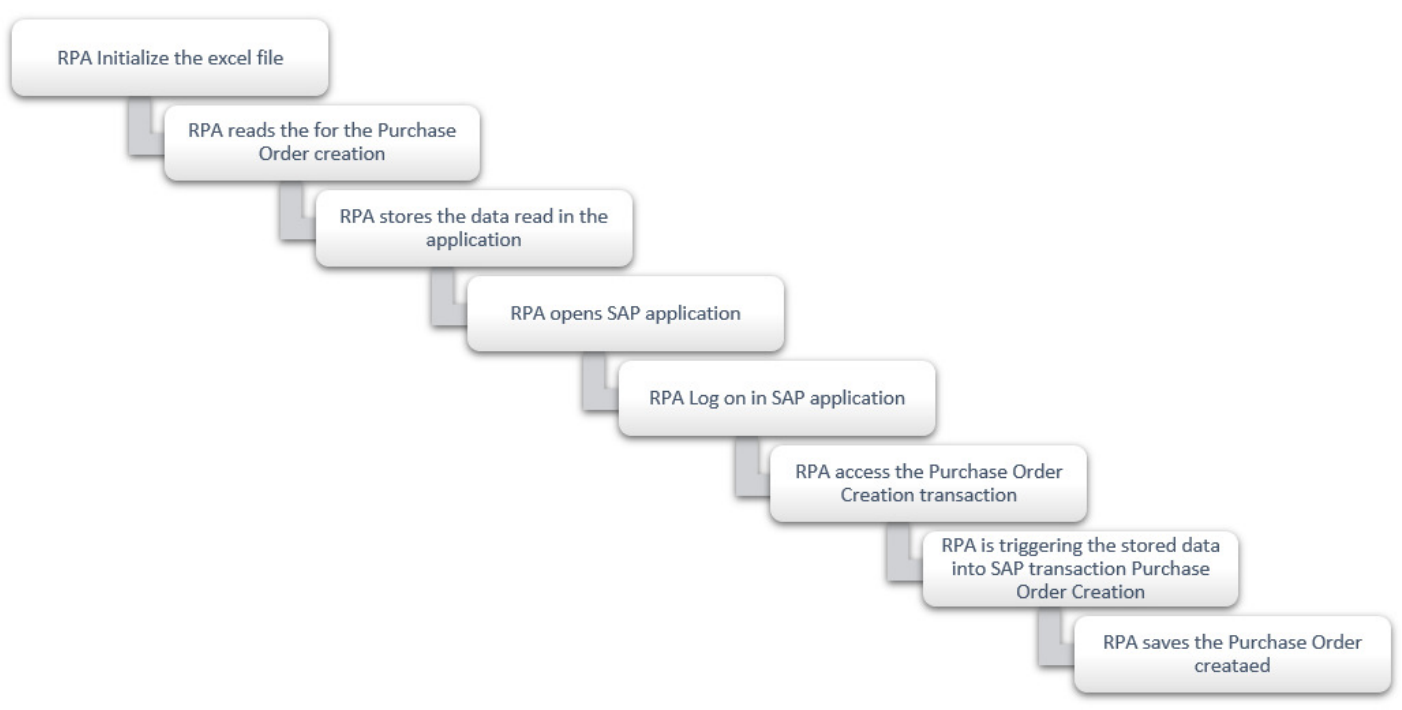

Fig 2. Scenario 1. Automatic order creation using SAP iRPA and integrating the SAP GUI interface.

There are several RPA platforms that are available in the market, including SAP SE [20] UiPath [21], Blue Prism [22] and Automation Anywhere [23]. For this work, we opted for the SAP iRPA solution, which offers a free platform for a limited time to build software robots. To demonstrate the use of SAP iRPA robots, this section is divided into two scenarios. The first scenario provides a demonstration of a software robot that performs the automatic creation of purchase orders by processing data from Microsoft Excel (see Figure 2).

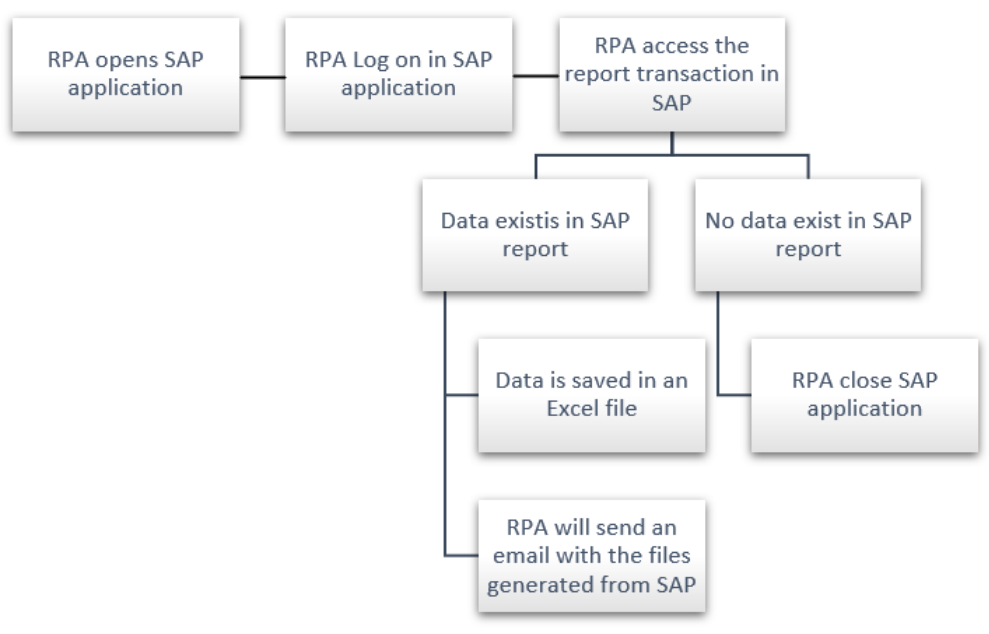

Fig 3. Report extraction and automatic generation of E-mails using SAP iRPA

Scenario two (Figure 3) provides an example of using a software robot to export relevant supply chain reports divided into multiple purchasing groups and informs the people responsible for each purchasing group by automatically generating E-mails. The scenarios provide a demonstration of the robots in action. In SAP iRPA, robots are

Elena PUICA, Journal of Supply Chain and Customer Relationship Management, 
created as a flowchart of events with interaction sequences. In the bot development phase, the necessary applications are captured and configured, workflows are defined, scripts are modified and much more. The applications to be automated must first be captured and configured; the next step is to create workflows by inserting activities that define how the bot will interact with the applications. An automation project in Desktop Studio includes declaring the application, defining the application, designing the workflow, entering the activities to run the Bot, and so on.

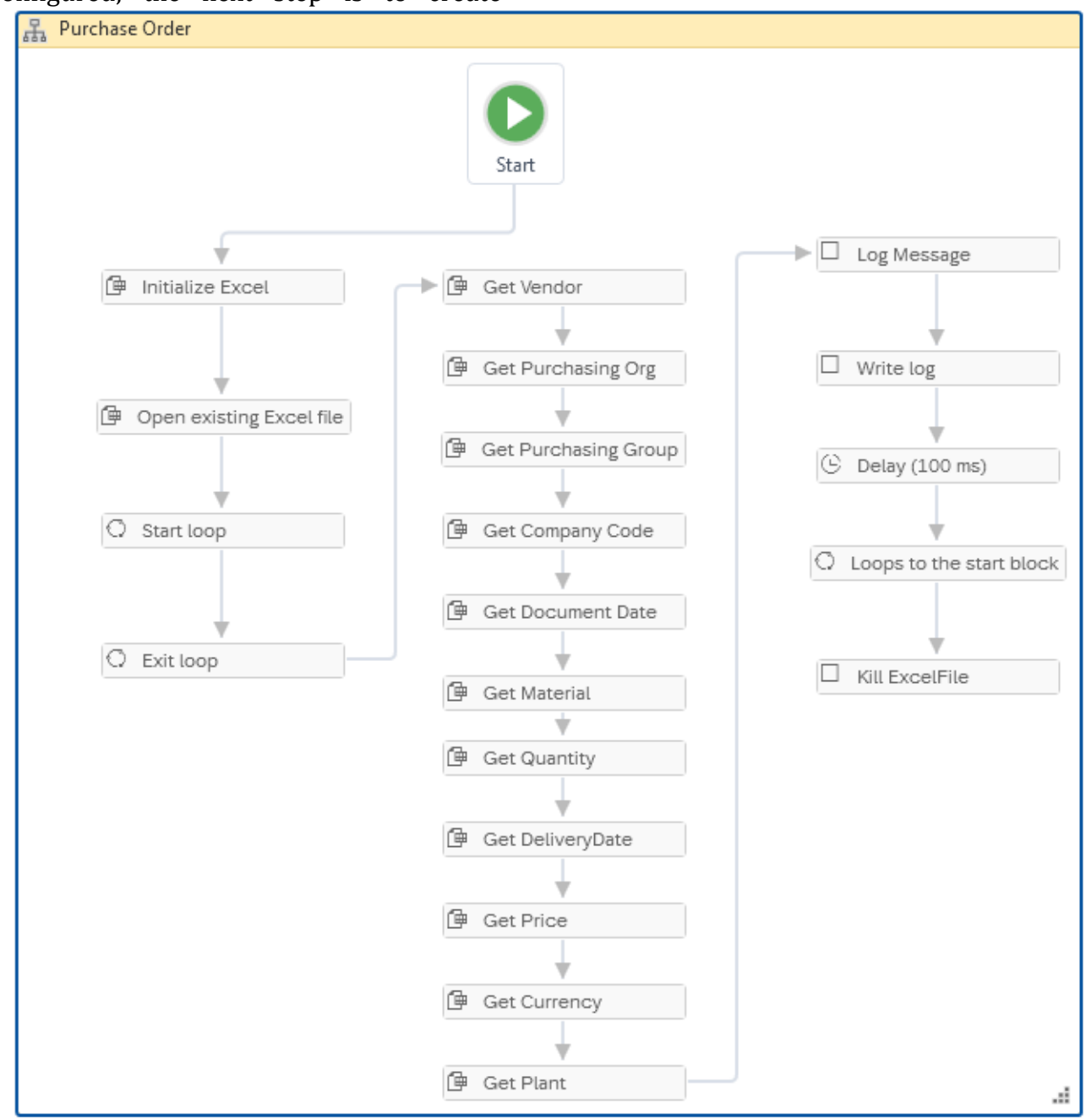

Fig 4. Scenario 1. Automatic order creation using SAP iRPA and integrating the SAP GUI interface. Step 2 - Workflow to extract data from Microsoft Excel.

Figure 4. represents the manipulation of data for their extraction from Microsoft Excel, so that these data are "read" by RPA, memorized into the application, and transferred in SAP system to create documents. The workflow starts from the initialization and opening of the Microsoft Excel application (the exact path of the file should be entered) and ends with the closing of this application. There are several possibilities that can be used as a data source for creating purchase orders in the computer system (for example, documents in PDF format). Loop activities should be added in the workflow, because the bots in this way should learn how to read the data

Elena PUICA, Journal of Supply Chain and Customer Relationship Management,

DOI: $10.5171 / 2022.221327$ 
for documents with more lines, or in case more documents should be created. The activity "Get" reads the data from Excel file and stores them into the application. This example can be utilized in many processes and tasks within SCM that involves an extraction of data from Microsoft Excel.

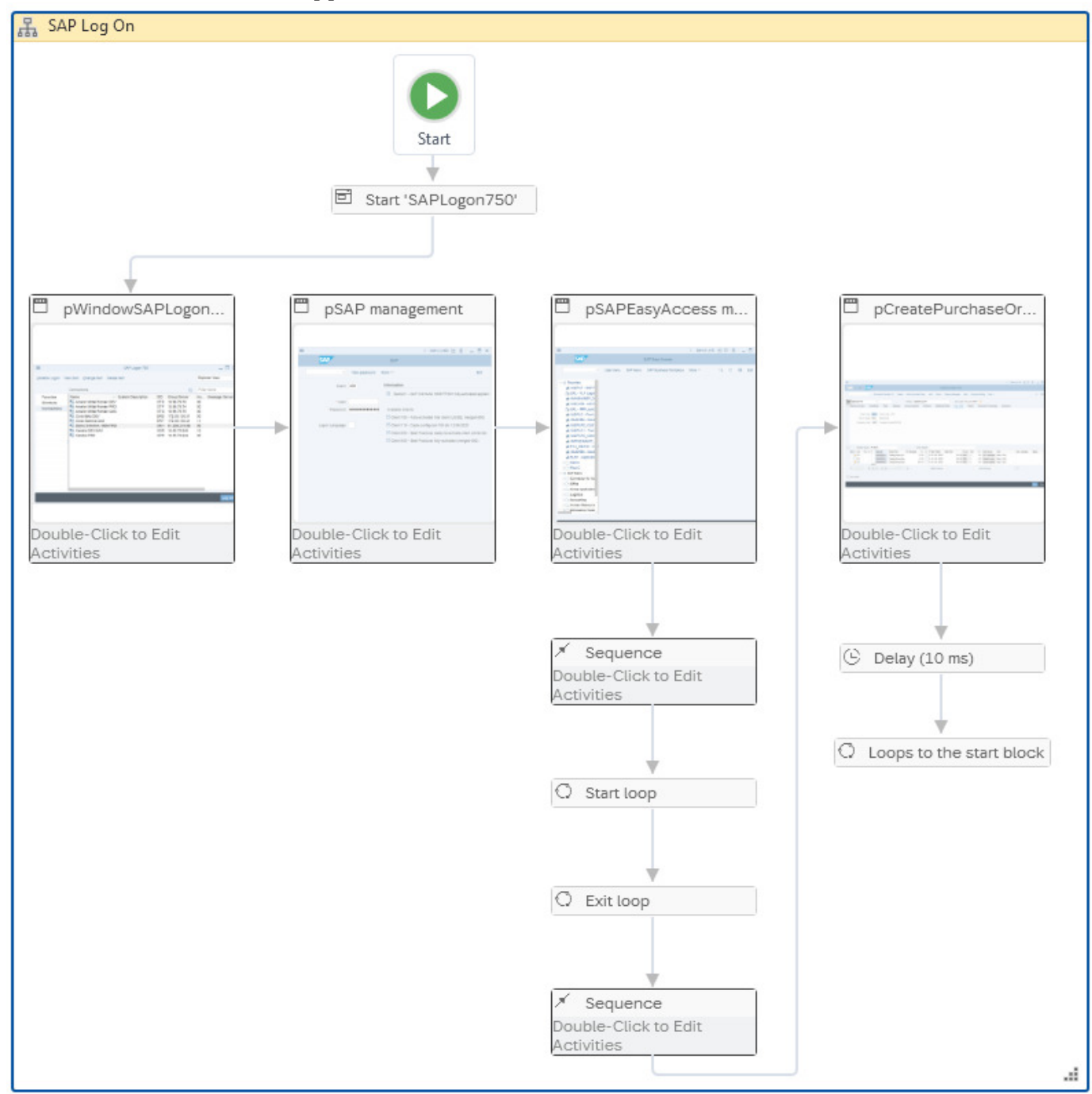

Fig 5. Scenario 1. Automatic order creation using SAP iRPA and integrating the SAP GUI interface. Step 1 - Workflow to create the purchase order.

Figure 5. is a representation of the activities positioned in the exact sequence that RPA will be triggered to create Purchase orders in SAP system, which is usually a process that needs repetitive work. By using RPA to automatically introduce the documents in the ERP system, the employees of procurement department can allocate more time to qualitative activities; for example, they can focus more on the relationship with suppliers. In just one month, there can be hundreds of orders to be processed in the information technology field, which can mean that the time allocated for their introduction of documents in ERP system is higher than the time allocated for high value activity. The workflow starts with an activity that is opening the SAP System and is followed by screens of SAP that are captured with iRPA. Each capture has its own activities and commands, so that the bot should know what are the tasks that 
should be triggered. The data are triggered from the data source where they are stored into the application based on Scenario 1,

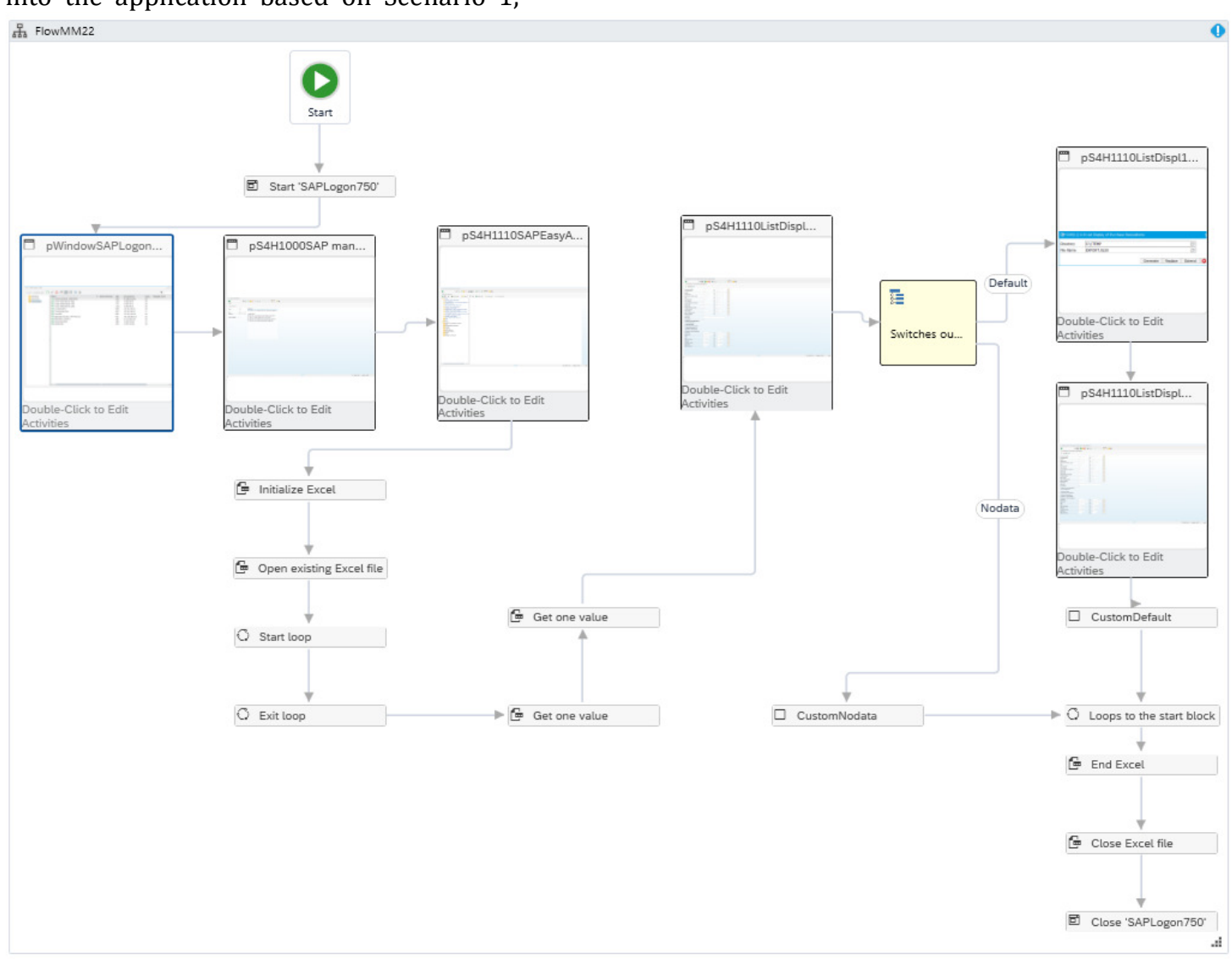

step 1 workflow to extract data from Microsoft Excel.

Fig 6. Scenario 2. Report extraction and automatic generation of E-mails using SAP iRPA

Figure 6. presents a scenario for extracting purchasing reports from the SAP system into Excel files. Employees can automatically receive personalized daily reports, depending on the department in which they are working, with the orders that have been confirmed or completed. The reports are received by E-mail and are extracted in Excel file format. Through this scenario, RPA is thought to trigger the commands to process the reports in the computer system and to send the reports to the representative workers, without having to access the computer system.

Both scenarios that were described are only a few representations on how iRPA can lead to reducing the manual and repetitive tasks within SCM. It has been shown how iRPA can be used to efficiently manage business

processes and to push workers to think of creative and better manners in their attributes, like strengthening the relationships with the suppliers, as well as expanding the SCM.

\section{Conclusion}

Organizations can apply RPA in SCM and pursue one or more objectives, such as process performance, efficiency, scalability, audibility, security, convenience, and compliance. However, organizations should consider both the advantages and disadvantages of implementing RPA, which can be an elaborate process. From the two scenarios provided, it was shown how iRPA can perform some of the routine and repetitive tasks in SCM. However, their implementation requires careful planning 
within the SCM, so that the digital environment is appropriate for its use. Among other things, iRPA can change the way organizations interact in the SCM. RPA is making its place in SCM more and more in many businesses and in major parts of the value chain. Most importantly, RPA has helped SCM to eliminate many levels of manual activities, but there are many changes that are needed to be implemented to fully get the benefit of RPA, more importantly, the changes will compel many enterprises to adopt new strategies. Also, enterprises need to adopt the way they do business, as there will be a transition from human operators to technology, enabled machines and robots. The trend in global industrial operation driven by RPA is exponentially increasing, which suggests RPA has either already become or is becoming a priority for many enterprises. All that had been said and based on the scenarios developed, all are pointing that RPA can have many benefits for SCM and its development.

\section{References}

- Alisha Asquith, Graeme Horsman (2019).Let the robots do it! - Taking a look at Robotic Process Automation and its potential application in digital forensics. School of Science, Engineering \& Design, Teesside University, Middlesbrough, Tees Valley, United Kingdom. http://doi.org/10.1016/j.fsir.2019.100 007

- Automation Anywhere, FUTUREPROOF YOUR RPA JOURNEY, (2019) Available https://www.automationanywhere.co $\mathrm{m} /$ (accessed at 06.11.2020)

- $\quad$ Blue Prism, Home, (2019) Available at: https://www.blueprism.com/ (accessed at 06.11.2020)

- Dwayne DeSylvia and Jonathan Yagos (2020). Introducing SAP® Intelligent Robotic Process Automation. Rheinwerk Publishing, 2020

- Fedor Kanakova, Igor Prokhorovb
(2020). Research and development of software robots for automating business processes of a commercial bank. Postproceedings of the 10th Annual International Conference on Biologically Inspired Cognitive Architectures, BICA 2019 (Tenth Annual Meeting of the BICA Society). 10.1016/j.procs.2020.02.196. CC BYNC-ND license (http://creativecommons.org/licenses /by-nc-nd/4.0/)

- Granta (11/12/17) Advantages and Disadvantages of Robotic Automation, Available at: https://www.grantaautomation.co.uk/news/advantagesand-disadvantagesofroboticautomation/

- IEEE Corporate Advisory Group. (2017). IEEE guide for terms and concepts in intelligent process automation.

- Jan vom Brocke, Wolfgang Maa, Peter Buxmann, Alexander Maedche, Jan Marco Leimeister, Günter Pecht (2018). Future Work and Enterprise Systems. Bus Inf Syst Eng 60(4):357366 https://doi.org/10.1007/s12599-0180544-2

- Jimenez-Ramirez, A., Reijers, H.A., Barba, I., Del Valle, C. (2019) A method to improve the early stages of the robotic process automation lifecycle. In: CAiSE

- Leno, V., Dumas, M., La Rosa, M., Maggi, F.M., Polyvyanyy, A. (2020) Automated discovery of data transformations for robotic process automation. In: AAAI IPA

- Leopold, H., van der Aa, H., Reijers, H.A. (2018) Identifying candidate tasks for robotic process automation in textual process descriptions. In: Enterprise, Business-Process and Information Systems Modeling

- MHI ANNUAL INDUSTRY REPORT. (2019). The 2019 MHI Annual Industry Report - Elevating Supply Chain Digital Consciousness. https://www.mhi.org/publications/re port\#download

Elena PUICA, Journal of Supply Chain and Customer Relationship Management, DOI: $10.5171 / 2022.221327$ 
- MHI ANNUAL INDUSTRY REPORT. (2020). The 2020 MHI Annual Industry Report - Embracing the Digital Mindset. https://www.mhi.org/publications/re port\#download

- Okoli, C. (2015). A guide to conducting a standalone systematic literature review. Communications of the Association for Information Systems, 37, 879-910. https://doi.org/10.17705/1CAIS.0374 3

- Peter Hofmann, Caroline Samp, Nils Urbach .(2019). Robotic process automation. Institute of Applied Informatics at University of Leipzig

- S. Madakam, M.R. Holmukhe, D. Kumar Jaiswal (2019). The future digital work force: Robotic Process Automation (RPA), (J. Inform. Syst. Technol. Manage. (2019) 16

- SAP SE, "Intelligent Robotic Process Automation", Available at: https://www.sap.com/products/robot ic-process-

automation.html?btp=c4156638-09aa411c-a8f1-ff45a4ac5110 (accessed at 06.11.2020)
- Sutherland, C. (2013). Framing a Constitution for Robotistan: Racing with the Machine of Robotic Automation : Hfs Research

- UiPath (n.d.), 'What is Robotic Process Automation?', Available at: https://www. uipath.com/rpa/roboticprocess-automation (accessed at 01.11.2020)

- UiPath (n.d.), 'What is Robotic Process Automation?', Available at: https://www.uipath.com/rpa/roboticprocess-automation (accessed at 06.11.2020)

- Vedder, R., \& Guynes, C. S. (2016) The challenge of botsourcing. Review of Business Information Systems (RBIS), $20(1)$. https://doi.org/10.19030/rbis.v20i1.9 677

- W.M. van der Aalst, M. Bichler, A. Heinzl (2018) Robotic process automation, (Bus. Inform. Syst. Eng. 60 (2018) 269

- Webster, J., \&Watson, R. T. (2002). Analyzing the past to prepare for the future: Writing a literature review. MIS Quarterly, 26, xiii-xxiii

Elena PUICA, Journal of Supply Chain and Customer Relationship Management, DOI: $10.5171 / 2022.221327$ 